\title{
Examen systématique du tétanos chez les personnes ayant des antécédents d'administration d'anatoxine tétanique
}

\author{
Hopkins J.P. ${ }^{1,2 *}$, Riddle C. ${ }^{3}$, Hollidge M. ${ }^{3}$, Wilson S.E. ${ }^{4,5}$ \\ ${ }^{1}$ Santé publique de la région de Niagara, Thorold, Ontario \\ ${ }^{2}$ Département d'épidémiologie clinique et de biostatistiques, Université McMaster de Hamilton, Ontario \\ ${ }^{3}$ Système de santé de Niagara, St. Catharines, Ontario \\ ${ }^{4}$ Santé publique Ontario, Toronto, Ontario \\ ${ }^{5}$ Dalla Lana School of Public Health, Université de Toronto, Toronto, Ontario \\ *Auteure-ressource : Jessica.hopkins@niagararegion.ca
}

\section{Résumé}

Objectifs : Évaluer les caractéristiques du tétanos chez des personnes qui ont déjà été vaccinées.

Méthodes : Une recherche systématique de la littérature a été menée dans les bases de données Ovid MEDLINE $^{\text {MD }}$ et EMBASE, pour les articles publiés entre 1946 et le 3 septembre 2013. La stratégie de recherche a été élaborée à l'aide de termes MeSH pour « tétanos », " immunisation » et " vaccination ». Les critères d'inclusion étaient les articles en anglais ou en français décrivant au moins un cas de tétanos, les antécédents de vaccination ou les résultats d'anticorps antitétaniques. Les articles ont été examinés pour les références pertinentes.

Résultats : 51 articles uniques publiés entre 1946 et 2013 ont été inclus dans l'examen. Les articles décrivaient 359 cas de tétanos clinique chez des personnes ayant déjà reçu une dose ou plus du vaccin contenant l'anatoxine tétanique ou présentant des concentrations de titres d'anticorps contre le tétanos généralement considérés comme ayant un effet protecteur. Des 210 cas ayant indiqué l'état du patient au moment de quitter l'hôpital, 180 (85,7 \%) ont survécu et seulement 3 cas présentaient des déficiences résiduelles.

Conclusion : Les spores du bacille tétanique sont omniprésentes et le présent rapport indique clairement que des cas de tétanos peuvent se produire chez les personnes ayant déjà reçu un vaccin contenant l'anatoxine tétanique. Les cliniciens ne devraient pas exclure le tétanos lorsque les symptômes cliniques semblent l'indiquer, quels que soient les antécédents de vaccination. Lorsque le tétanos est traité, son pronostic est bon. Des recherches plus approfondies sont nécessaires pour évaluer l'incidence du tétanos dans les populations partiellement ou entièrement immunisées et pour déterminer si cela est attribuable à une diminution de l'immunité en raison de l'échec de l'immunisation.

\section{Introduction}

Un homme de 22 ans auparavant en bonne santé s'est présenté à un service d'urgence en Ontario, au Canada avec des symptômes de spasmes et de trismus conformes au tétanos. II a signalé une blessure mineure au gros orteil gauche qui semblait former un abcès et qui était survenue vingt-sept jours avant de se présenter. II a incisé l'abcès lui-même, mais la plaie s'est aggravée et il a alors tenté d'obtenir un traitement médical. Le traitement médical comprenait la prise d'antibiotiques, puis la prise de stéroïdes systémiques pour une réaction allergique soupçonnée aux antibiotiques. Les antécédents médicaux ont révélé cinq doses documentées et espacées de façon appropriée d'un vaccin contenant l'anatoxine tétanique : diphtérie, tétanos, coqueluche (DTC) à l'âge de 2 , 4 et 6 mois, diphtérie, tétanos, coqueluche acellulaire, virus de la polio inactivé (DCaT-VPI) à l'âge de 18 mois et tétanos, diphtérie (dose réduite), coqueluche acellulaire (dose réduite) (dcaT) à l'âge de 14 ans, 9 ans auparavant. II n'y avait aucune preuve de rappel de vaccin à l'âge préscolaire, généralement administré entre 4 et 6 ans. Aucun autre vaccin contenant l'anatoxine tétanique n'a été administré lorsqu'il a initialement tenté d'obtenir un traitement médical. À l'hôpital, le patient a été traité par immunoglobuline antitétanique, des antibiotiques et 
des soins de soutien. Durant son séjour à l'hôpital, l'état du patient s'est amélioré et il a quitté l'hôpital 20 jours après son admission, avec un rétablissement complet signalé 12 semaines après sa présentation initiale.

Le tétanos est la manifestation clinique de l'infection à Clostridium tetani (1). L'exotoxine produite par le bacille tétanique agit sur la moelle épinière et cause de douloureuses contractions musculaires, en particulier de la nuque et du muscle masséter, d'où le nom familier anglais de « lockjaw » (mâchoire bloquée) (2). Les symptômes plus graves comprennent des problèmes respiratoires, le coma et la mort (2). Les spores du bacille tétanique sont omniprésentes dans l'environnement et peuvent infecter toute plaie exposée (1). On prévient le tétanos par des soins appropriés de la plaie et l'immunisation (1).

Le tétanos est rare au Canada, avec une moyenne de quatre cas par année (intervalle 1-10 cas par année) entre 1990 et 2010 (3). Depuis les années 1920, on a observé une diminution importante du nombre de décès liés au tétanos en raison de l'accessibilité du vaccin et de l'amélioration des soins intensifs $(1,2)$. Le taux de létalité en raison du tétanos chez les personnes non vaccinées varie beaucoup, allant de $10 \%$ à plus de $80 \%$; les personnes très jeunes et les personnes âgées sont celles qui présentent le plus grand risque $(1,3,4)$.

Au Canada, le calendrier d'immunisation systématique comprend quatre doses du vaccin contenant l'anatoxine tétanique, administré à l'âge de 2, 4 et 6 mois et entre l'âge de 12 et 23 mois (habituellement à 18 mois), ainsi qu'une dose de rappel entre l'âge de 4 et 6 ans (3). Après l'administration des trois premières doses d'un vaccin contenant l'anatoxine tétanique, plus de $99 \%$ des personnes présentent des preuves de titres d'anticorps protecteurs (3). Même si, par le passé, les titres d'anticorps contre le tétanos supérieurs à $0,01 \mathrm{Ul} / \mathrm{mL}$ par réaction de neutralisation chez les souris ont été considérés comme protecteurs, certaines études ont indiqué la nécessité d'indicateurs de protection plus élevés, tels que $0,1 \mathrm{UI} / \mathrm{mL}$ (5-7). Des études d'observation ont mis en évidence l'efficacité des régimes d'immunisation avant et après l'exposition d'une blessure (3). Des doses de rappel subséquentes ont été recommandées à intervalles de 10 ans, même si la version la plus récente du Guide canadien d'immunisation indique que de nouvelles données probantes sur le moment d'administration optimal des doses de rappel font actuellement l'objet d'un examen (3). Selon la nature de la plaie et les antécédents de vaccination, une immunisation après l'exposition (active et passive) peut également être indiquée (3).

Quoi qu'il en soit, le tétanos peut quand même survenir après l'immunisation. Compte tenu du cas de tétanos cidessus ayant des antécédents d'une série primaire complète et documentée d'administration d'anatoxine tétanique, ainsi qu'une dose de rappel administrée neuf ans avant la présentation à l'hôpital, un examen systématique a été effectué pour évaluer les caractéristiques du tétanos chez les personnes qui ont déjà été vaccinées.

\section{Méthodes}

Une recherche systématique de la littérature a été menée dans les bases de données Ovid MEDLINE ${ }^{\mathrm{MD}}$ et EMBASE, pour les articles publiés entre 1946 et le 3 septembre 2013. La stratégie de recherche a été conçue dans MEDLINE en utilisant les termes MeSH suivants : tétanos/, anatoxine tétanique/, diagnostic/, diagnostic différentiel/, immunisation/, vaccination/. Le vocabulaire normalisé a été complété par l'utilisation de mots clés connexes afin d'accroître la spécificité de la recherche : " entièrement », " préexistant », " antécédent* », " avant », " présen* », « protecteur », « actif », « anticorps », « immuno* », " vaccin* ». Les termes de recherche ont été combinés à l'aide d'opérateurs booléens. Aucune limite n'a été appliquée pour une recherche dans MEDLINE. Après la traduction du vocabulaire normalisé en termes Emtree, la recherche dans EMBASE a été limitée au contenu qui n'était pas dans MEDLINE.

On a examiné les résumés pour conserver les articles écrits en anglais ou en français contenant un diagnostic de tétanos chez au moins une personne. Les résumés respectant les deux critères de sélection ont été retenus pour l'examen en texte intégral. Deux auteurs (J.P.H. et S.E.W.) ont passé en revue les articles aux fins d'inclusion dans l'examen. 
Pour être inclus dans l'examen, l'article devait décrire au moins un cas de tétanos, les antécédents de vaccination d'un cas entièrement ou partiellement immunisé ou présentant des résultats d'anticorps antitétaniques et être écrit en anglais ou en français. La recherche formelle a été complétée par des recherches en boule de neige dans PubMed sur les articles respectant les critères d'inclusion prédéterminés. En outre, un examen des références provenant de chaque article pertinent a été effectué.

Les données pertinentes, y compris le nombre de cas de tétanos, l'âge, le sexe, les titres d'anticorps, le résultat clinique, les antécédents d'immunisation comprenant l'anatoxine tétanique, y compris le nombre de doses et les délais, ont été extraits et regroupées (Microsoft Excel 2010, Redmond, Washington). Un auteur (J.P.H.) a résumé les données examinées aux fins d'exactitude par un deuxième auteur (S.E.W.). Les désaccords (qui étaient rares) ont été réglés par un consensus.

Une évaluation formelle de la qualité de chacun des articles n'a pas été effectuée. Les articles récupérés comprenaient des rapports des cas, des études de séries de cas ou des rapports de surveillance. À la connaissance des auteurs, il n'existe pas d'outil validé d'évaluation de la qualité de ces modèles d'étude. Toutefois, les études qui n'avaient pas de données pertinentes ont été exclues, conformément aux critères a priori d'inclusion et d'exclusion. Comme il s'agissait d'une synthèse descriptive de la documentation, les graphiques en entonnoir et les évaluations statistiques de l'hétérogénéité n'étaient pas des mesures appropriées de biais de publication.

\section{Résultats}

Plus de 4000 articles ont été déterminés initialement. Cinquante et un articles uniques ont été inclus dans l'examen. La Figure 1 résume les résultats de la recherche de la documentation.

Figure 1 : Résultats de la recherche de la documentation

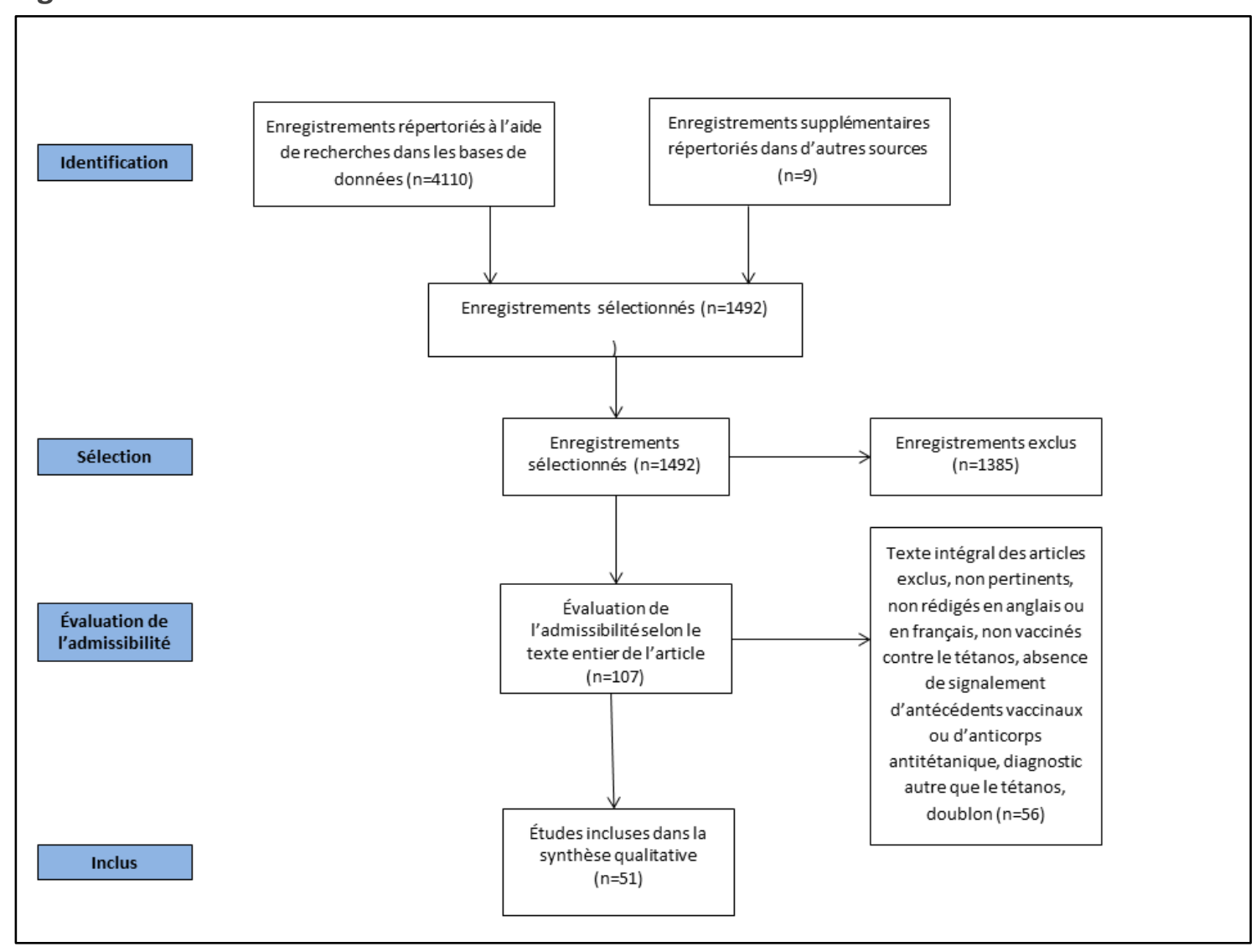


Dans les 51 études incluses, on comptait 359 cas de tétanos clinique chez des personnes ayant déjà reçu une dose ou plus du vaccin comprenant l'anatoxine tétanique ou présentant des concentrations de titres d'anticorps contre le tétanos généralement considérés comme protecteurs (8-9). La majorité des études $(n=25)$ étaient fondées sur des données provenant des États-Unis. Des 47 cas dont l'âge et le sexe étaient décrits, $26(39,4 \%)$ étaient des hommes d'âge médian de 26 ans (intervalle 1-79 ans). On a analysé les titres d'anticorps contre le tétanos de 14 cas avant l'administration d'anatoxine tétanique, tandis que les 345 autres cas présentaient une immunisation confirmée par l'examen du dossier de vaccination. Tous les cas de tétanos ont été diagnostiqués en fonction des signes et des symptômes cliniques compatibles. L'isolement de Clostridium tetani n'a été signalé dans aucun cas.

Les antécédents de vaccination des cas ont été examinés dans les rapports de cas. Même si les critères d'inclusion exigeaient que tous les cas aient reçu au moins une dose, seuls 175 (48,7\%) cas ont signalé le nombre exact de doses. Quatre-vingt-quatorze cas $(26,2 \%$ du nombre total de cas) avaient reçu trois doses ou plus, ce qui indique que la série primaire pouvait avoir été réalisée, même s'il n'était pas possible d'évaluer l'intervalle entre les doses. En ce qui concerne les doses administrées au-delà de la petite enfance et décrites comme des doses de rappel, mais qui peuvent avoir été précédées ou non d'une série complète d'immunisations primaires, 57 cas $(15,9 \%)$ ont reçu une dose de rappel de l'anatoxine tétanique au cours des dix dernières années, 54 cas $(15,0 \%)$ ont reçu une dose de rappel dix ans ou plus auparavant et 248 cas $(69,1 \%)$ ont reçu une dernière dose de rappel à un intervalle inconnu (ou n'ont reçu aucune dose de rappel), ou le cas n'était pas admissible à une dose de rappel (p. ex. en fonction d'une politique d'antécédents ou d'âge) (données non indiquées). Étant donné que plus de la moitié des cas n'ont pas signalé le nombre de doses du vaccin comprenant l'anatoxine tétanique reçues et qu'on a observé un petit nombre de décès $(n=30)$, il n'a pas été possible d'analyser les données de survie en fonction du nombre de doses.

Le taux de survie élevé dans les rapports de cas publiés semblent indiquer une maladie plus bénigne qui est associée à un meilleur pronostic chez les personnes ayant des antécédents d'immunisation antitétanique $(3,4$, 20-23). Cela est conforme à une atténuation de la gravité clinique chez les hôtes vaccinés qui est reconnue dans d'autres maladies évitables par la vaccination (24-26).

Des 180 de 210 cas $(85,7 \%)$ ayant signalé les résultats cliniques qui ont survécu après leur congé de l'hôpital et parmi les cas qui ont fait l'objet d'un suivi après leur congé de l'hôpital, tous sauf trois $(17,27,28)$ présentaient une résolution complète des symptômes. Même si le taux de survie semblait généralement s'améliorer au fil du temps, $42 \%$ des études $(n=149)$ n'ont pas signalé le résultat clinique. II n'a donc pas été possible d'étudier les tendances de survie étant donné qu'elles sont liées à d'autres facteurs ( $p$. ex. améliorations systématiques des soins intensifs au fil du temps et augmentation de la mise en œuvre de séries d'immunisations primaires à 3 doses). Les études sont résumées dans les Tableaux 1 et 2.

\section{Tableau 1 : Rapports de cas de tétanos chez des personnes présentant des données complètes} (renseignements détaillés sur les antécédents de vaccination contre le tétanos ou sur les anticorps contre le tétanos et sur les résultats cliniques)

\begin{tabular}{l|l|c|l|l|l|l} 
Auteur / pays & $\begin{array}{l}\text { Plan d'étude } \\
\text { I nombre de } \\
\text { cas (n) }\end{array}$ & $\begin{array}{l}\text { Âge (ans) } \\
\text { et sexe }\end{array}$ & $\begin{array}{l}\text { Facteurs } \\
\text { de risque } \\
\text { du tétanos }\end{array}$ & $\begin{array}{l}\text { Antécédents } \\
\text { de } \\
\text { vaccination }\end{array}$ & $\begin{array}{l}\text { Titre } \\
\text { d'anticorps } \\
\text { contre le } \\
\text { tétanos } \\
\text { lors du } \\
\text { diagnostic }^{4}\end{array}$ & $\begin{array}{l}\text { Résultat } \\
\text { pour le } \\
\text { patient }\end{array}$ \\
\hline $\begin{array}{l}\text { Abrahamian (8)/ } \\
\text { États-Unis }\end{array}$ & $\begin{array}{l}\text { Rapport de } \\
\text { cas } / \mathrm{n}=1\end{array}$ & $45 \mathrm{H}$ & UDI & $?$ & ++ & - \\
\hline $\begin{array}{l}\text { Atabek (9) / } \\
\text { Turquie }\end{array}$ & $\begin{array}{l}\text { Rapport de } \\
\text { cas } / \mathrm{n}=1\end{array}$ & $7 \mathrm{~F}$ & Lacération & ++ & $?$ & + \\
\hline $\begin{array}{l}\text { Aydin-Teke (29) } \\
\text { / Turquie }\end{array}$ & $\begin{array}{l}\text { Rapport de } \\
\text { cas } / \mathrm{n}=1\end{array}$ & $15 \mathrm{H}$ & Blessures & ++ & $?$ & +
\end{tabular}




\begin{tabular}{|c|c|c|c|c|c|c|}
\hline $\begin{array}{l}\text { Bardenheier (22 } \\
\text { ) / États-Unis }\end{array}$ & $\begin{array}{c}\text { Surveillance } / \\
n=31\end{array}$ & $?$ & $?$ & $\begin{array}{l}+(15) \\
++(16)\end{array}$ & $?$ & $\begin{array}{l}+(30) \\
-(1)\end{array}$ \\
\hline $\begin{array}{l}\text { Berger }(16) / \\
\text { États-Unis }\end{array}$ & $\begin{array}{l}\text { Rapport de } \\
\text { cas } / \mathrm{n}=1\end{array}$ & $25 \mathrm{~F}$ & UDI & $?$ & + & + \\
\hline $\begin{array}{l}\text { Boyd (30) / } \\
\text { Europe et } \\
\text { Afrique }\end{array}$ & $\begin{array}{c}\text { Surveillance } \\
\text { rétrospective / } \\
\mathrm{n}=16\end{array}$ & $?$ & $?$ & $+/++$ & $?$ & $\begin{array}{l}+(11) \\
-(5)\end{array}$ \\
\hline $\begin{array}{l}\text { Boyer (31)/ } \\
\text { France }\end{array}$ & $\begin{array}{l}\text { Examen de } \\
\text { cas } / \mathrm{n}=10\end{array}$ & $?$ & $?$ & $+/++$ & $?$ & $\begin{array}{l}+(2) \\
-(8)\end{array}$ \\
\hline $\begin{array}{l}\text { Coniglione (32) / } \\
\text { États-Unis }\end{array}$ & $\begin{array}{l}\text { Rapport de } \\
\text { cas } / \mathrm{n}=1\end{array}$ & $29 \mathrm{H}$ & Blessures & ++ & ++ & + \\
\hline $\begin{array}{l}\text { Crone (17) / } \\
\text { États-Unis }\end{array}$ & $\begin{array}{l}\text { Rapport de } \\
\text { cas } / n=3\end{array}$ & $\begin{array}{l}29 \mathrm{H} \\
42 \mathrm{~F} \\
57 \mathrm{~F}\end{array}$ & $\begin{array}{c}\text { ? } \\
\text { UDI } \\
\text { Seringue } \\
\text { réutilisée }\end{array}$ & $\begin{array}{c}++ \\
+ \\
+\end{array}$ & $++(3)$ & $\begin{array}{c}+ \\
- \\
+ \\
\text { (déficiences) }\end{array}$ \\
\hline $\begin{array}{l}\text { de la } \\
\text { Chapelle (33) / } \\
\text { France }\end{array}$ & $\begin{array}{l}\text { Rapport de } \\
\text { cas } / \mathrm{n}=1\end{array}$ & $52 \mathrm{H}$ & Blessure, IS & $?$ & ++ & + \\
\hline $\begin{array}{l}\text { Dyce }(27) / \\
\text { États-Unis }\end{array}$ & $\begin{array}{l}\text { Rapport de } \\
\text { cas } / \mathrm{n}=1\end{array}$ & $24 \mathrm{~F}$ & Perçage & ++ & $?$ & $\begin{array}{c}+ \\
\text { (déficiences) } \\
\end{array}$ \\
\hline $\begin{array}{l}\text { Faust (34) / } \\
\text { États-Unis }\end{array}$ & $\begin{array}{c}\text { Surveillance } \\
\text { des hôpitaux / } \\
n=1\end{array}$ & $5 \mathrm{H}$ & $?$ & + & $?$ & + \\
\hline $\begin{array}{l}\text { Fiorillo (35) / } \\
\text { Canada }\end{array}$ & $\begin{array}{l}\text { Rapport de } \\
\text { cas } / \mathrm{n}=1\end{array}$ & $10 \mathrm{H}$ & Blessure & ++ & ++ & + \\
\hline $\begin{array}{l}\text { Hall (36) / États- } \\
\text { Unis }\end{array}$ & $\begin{array}{l}\text { Rapport de } \\
\text { cas } / n=2\end{array}$ & $?$ & $?$ & $+(2)$ & $?(2)$ & $\begin{array}{l}+(1) \\
-(1)\end{array}$ \\
\hline $\begin{array}{l}\text { Hedrick (37) / } \\
\text { États-Unis }\end{array}$ & $\begin{array}{l}\text { Rapport de } \\
\text { cas } / \mathrm{n}=2\end{array}$ & $\begin{array}{l}10 \mathrm{H} \\
45 \mathrm{H}\end{array}$ & $\begin{array}{c}\text { Blessure } \\
?\end{array}$ & $\begin{array}{c}++ \\
+\end{array}$ & $?(2)$ & $+(2)$ \\
\hline $\begin{array}{l}\text { Hopkins (le } \\
\text { présent rapport) } \\
\text { / Canada }\end{array}$ & $\begin{array}{l}\text { Rapport de } \\
\text { cas } / \mathrm{n}=1\end{array}$ & $22 \mathrm{H}$ & Blessure, IS & ++ & $?$ & + \\
\hline $\begin{array}{l}\text { König (10) / } \\
\text { Allemagne }\end{array}$ & $\begin{array}{l}\text { Rapport de } \\
\text { cas } / n=1\end{array}$ & $14 \mathrm{H}$ & Blessure & ++ & $?$ & + \\
\hline $\begin{array}{l}\text { Livorsi (11) / } \\
\text { États-Unis }\end{array}$ & $\begin{array}{l}\text { Rapport de } \\
\text { cas } / \mathrm{n}=1\end{array}$ & $44 \mathrm{H}$ & Blessure & $?$ & ++ & + \\
\hline $\begin{array}{l}\text { Lodha (38) / } \\
\text { Inde }\end{array}$ & $\begin{array}{l}\text { Rapport de } \\
\text { cas } / \mathrm{n}=2\end{array}$ & $\begin{array}{c}3 \mathrm{~F} \\
4,5 \mathrm{H}\end{array}$ & $?(2)$ & $\begin{array}{c}? ? \\
++\end{array}$ & $?(2)$ & $+(2)$ \\
\hline $\begin{array}{l}\text { Long (39) / } \\
\text { États-Unis }\end{array}$ & $\begin{array}{c}\text { Surveillance / } \\
n=6\end{array}$ & $?$ & Blessure & $++(6)$ & $?$ & $\begin{array}{l}+(3) \\
-(3)\end{array}$ \\
\hline $\begin{array}{l}\text { Loscalzo (40) / } \\
\text { États-Unis }\end{array}$ & $\begin{array}{l}\text { Rapport de } \\
\text { cas } / \mathrm{n}=1\end{array}$ & $23 \mathrm{~F}$ & Perçage & ++ & $?$ & + \\
\hline $\begin{array}{l}\text { Luisto (12) / } \\
\text { Finlande }\end{array}$ & $\begin{array}{c}\text { Série de cas } \\
\text { rétrospective / } \\
n=5\end{array}$ & $\begin{array}{l}5 \mathrm{H} \\
10 \mathrm{~F} \\
12 \mathrm{H} \\
13 \mathrm{~F} \\
15 \mathrm{~F}\end{array}$ & $\begin{array}{l}\text { Morsure } \\
\text { d'animal } \\
\text { Infection } \\
\text { Blessure } \\
\text { Blessure } \\
\text { Brûlure }\end{array}$ & $\begin{array}{c}++ \\
++ \\
++ \\
++ \\
?\end{array}$ & $?(5)$ & $+(5)$ \\
\hline $\begin{array}{l}\text { Newton- } \\
\text { John (23) / } \\
\text { Australie }\end{array}$ & $\begin{array}{l}\text { Séries de cas } \\
\qquad / \mathrm{n}=19\end{array}$ & $?$ & $?$ & $\begin{array}{l}++(13) \\
+(6)\end{array}$ & $?$ & $\begin{array}{l}+(17) \\
-(2)\end{array}$ \\
\hline $\begin{array}{l}\text { Otero- } \\
\text { Maldonado (28) } \\
\text { / Porto Rico }\end{array}$ & $\begin{array}{c}\text { Rapport de } \\
\text { cas et } \\
\text { surveillance / } \\
n=7\end{array}$ & $\begin{array}{l}67 \mathrm{H} \\
?(6)\end{array}$ & $\begin{array}{c}\text { Blessure } \\
?(6)\end{array}$ & $\begin{array}{l}+ \\
?(6)\end{array}$ & $?(7)$ & $\begin{array}{c}+ \text { (déficience } \\
\text { s) } \\
+(5),-(1)\end{array}$ \\
\hline $\begin{array}{l}\text { Pascual (21) / } \\
\text { États-Unis }\end{array}$ & $\begin{array}{c}\text { Surveillance / } \\
n=30\end{array}$ & $?$ & $?$ & $\begin{array}{l}+(10) \\
++(20)\end{array}$ & $?(30)$ & $\begin{array}{c}+(10) \\
+(19),-(1) \\
\end{array}$ \\
\hline Passen (14) / & Rapport de & $35 \mathrm{H}$ & Blessure & ++ & ++ & + \\
\hline
\end{tabular}




\begin{tabular}{|c|c|c|c|c|c|c|}
\hline États-Unis & cas $/ n=1$ & & & & & \\
\hline $\begin{array}{l}\text { Peterson (18) / } \\
\text { Suède }\end{array}$ & $\begin{array}{l}\text { Rapport de } \\
\text { cas } / \mathrm{n}=1\end{array}$ & $12 \mathrm{H}$ & Blessure & ++ & ++ & + \\
\hline $\begin{array}{l}\text { Spittle (41)/ } \\
\text { Nouvelle- } \\
\text { Zélande }\end{array}$ & $\begin{array}{l}\text { Rapport de } \\
\text { cas } / \mathrm{n}=1\end{array}$ & $25 \mathrm{~F}$ & Blessure & ++ & $?$ & + \\
\hline $\begin{array}{l}\text { Tiwari (4) / } \\
\text { États-Unis }\end{array}$ & $\begin{array}{c}\text { Surveillance / } \\
n=55\end{array}$ & $?$ & $?$ & $\begin{array}{l}+(26) \\
++(29)\end{array}$ & $?$ (55) & $\begin{array}{c}+(17),-(3), \\
?(6) \\
+(24),-(1) \\
?(4)\end{array}$ \\
\hline $\begin{array}{l}\text { Vieria (19) / } \\
\text { Australie } \\
\text { ne), } \mathrm{F} \text { (femme), ? (in } \\
\text { nu / non indiqué) } \\
\text { ses ou plus), + (1 ou } \\
\mathrm{Ul} / \mathrm{mL}),+(0,01-0,0 \\
\text { au moment de quitt } \\
\text { lisateur de drogues ir } \\
\text { unosuppression }\end{array}$ & $\begin{array}{l}\text { Rapport de } \\
\text { cas / } \mathrm{n}=1 \\
\text { nnu / non indiqué) } \\
\text { doses), ? (nombre } \\
\text { II/mL), ? = non mes } \\
\text { l'hôpital), - (décédé } \\
\text { ctables }\end{array}$ & $18 \mathrm{H}$ & $\begin{array}{l}\text { indiqué) } \\
\text { Idiqué) }\end{array}$ & ++ & $?$ & + \\
\hline
\end{tabular}


Tableau 2 : Rapports de cas de tétanos chez des personnes ne présentant pas de données complètes (renseignements détaillés sur les antécédents de vaccination contre le tétanos ou résultat clinique)

\begin{tabular}{|c|c|c|c|c|c|c|}
\hline Auteur / pays & $\begin{array}{c}\text { Plan d'étude / } \\
\text { nombre de } \\
\text { cas }(n)\end{array}$ & $\begin{array}{l}\text { Âge (ans) } \\
\text { et sexe }\end{array}$ & $\begin{array}{c}\text { Facteurs } \\
\text { de risque } \\
\text { du } \\
\text { tétanos }\end{array}$ & $\begin{array}{c}\text { Antécédents } \\
\text { de } \\
\text { vaccination }^{3}\end{array}$ & $\begin{array}{c}\text { Titre } \\
\text { d'anticorps } \\
\text { contre le } \\
\text { tétanos } \\
\text { lors du } \\
\text { diagnostic }\end{array}$ & $\begin{array}{l}\text { Résultat } \\
\text { pour le } \\
\text { patient }^{5}\end{array}$ \\
\hline $\begin{array}{l}\text { Bankole (42) / } \\
\text { Nigéria }\end{array}$ & $\begin{array}{c}\text { Séries de cas / } \\
n=11\end{array}$ & $?$ & $?$ & $?$ & $?$ & $?$ \\
\hline $\begin{array}{l}\text { Beltran (43)) / États- } \\
\text { Unis }\end{array}$ & $\begin{array}{c}\text { Rapport de cas } \\
/ \mathrm{n}=1\end{array}$ & $58 \mathrm{H}$ & $\begin{array}{l}\text { Morsure } \\
\text { d'animal }\end{array}$ & $?$ & ++ & $?$ \\
\hline $\begin{array}{l}\text { Bunch (44) / États- } \\
\text { Unis }\end{array}$ & $\begin{array}{l}\text { Séries de cas / } \\
\quad n=5\end{array}$ & $\begin{array}{l}53 \mathrm{~F} \\
59 \mathrm{~F} \\
62 \mathrm{~F} \\
65 \mathrm{~F} \\
75 \mathrm{~F}\end{array}$ & $\begin{array}{c}\text { ? } \\
\text { Blessure } \\
\text { Infection } \\
\text { Lacération } \\
\text { Lacération }\end{array}$ & $?(5)$ & $?(5)$ & $+(5)$ \\
\hline $\begin{array}{l}\text { Christensen (45) / } \\
\text { États-Unis }\end{array}$ & $\begin{array}{c}\text { Rapport de cas } \\
\qquad / \mathrm{n}=1\end{array}$ & $10 \mathrm{H}$ & Blessure & + & $?$ & $?$ \\
\hline $\begin{array}{l}\text { Culbertson (46) / } \\
\text { États-Unis }\end{array}$ & $\begin{array}{c}\text { Rapport de cas } \\
/ \mathrm{n}=1\end{array}$ & $41 \mathrm{H}$ & $\begin{array}{c}\text { Brûlure, } \\
\text { lacérations }\end{array}$ & $?$ & $?$ & + \\
\hline deSouza (47) / Inde & $\begin{array}{c}\text { Cas témoin / } \\
\mathrm{n}=1\end{array}$ & $?$ & $?$ & + & $?$ & $?$ \\
\hline $\begin{array}{l}\text { Earis (48) / } \\
\text { Royaume-Uni }\end{array}$ & $\begin{array}{l}\text { Rapport de cas } \\
\qquad / \mathrm{n}=1\end{array}$ & $66 \mathrm{~F}$ & $\begin{array}{c}\text { Tumeur } \\
\text { fongique }\end{array}$ & $?$ & $?$ & + \\
\hline $\begin{array}{l}\text { Edsall (49) / } \\
\text { multiples }\end{array}$ & $\begin{array}{c}\text { Examen des } \\
\text { cas publiés } \\
\text { précédemment }{ }^{6} / \\
n=4\end{array}$ & $?$ & $?$ & $?(4)$ & $?$ & $\begin{array}{l}+(3) \\
-(1)\end{array}$ \\
\hline $\begin{array}{l}\text { Ferris (50) / } \\
\text { Royaume-Uni }\end{array}$ & $\begin{array}{l}\text { Rapport de cas } \\
/ \mathrm{n}=1\end{array}$ & $17 \mathrm{H}$ & Trauma & $?$ & $?$ & + \\
\hline Geeta (51) / Inde & $\begin{array}{c}\text { Séries de cas / } \\
n=12\end{array}$ & $\begin{array}{c}1 \mathrm{H} \\
?(11) \\
\end{array}$ & $?$ & $\begin{array}{c}++ \\
+(11) \\
\end{array}$ & $?$ & $?$ \\
\hline $\begin{array}{l}\text { Hahn (52) / États- } \\
\text { Unis }\end{array}$ & $\begin{array}{c}\text { Rapport de cas } \\
/ \mathrm{n}=1\end{array}$ & $58 \mathrm{H}$ & $?$ & $?$ & $?$ & + \\
\hline $\begin{array}{l}\text { Henderson (53) / } \\
\text { États-Unis }\end{array}$ & $\begin{array}{l}\text { Séries de cas / } \\
\qquad n=5\end{array}$ & $?$ & $\begin{array}{c}\text { Différentes } \\
\text { blessures; } \\
\text { UDI }\end{array}$ & $?$ & $?$ & $?$ \\
\hline Iqbal (54) / Pakistan & $\begin{array}{c}\text { Séries de cas / } \\
n=10\end{array}$ & $?$ & $?$ & $?$ & $?$ & $?$ \\
\hline Lee(55) / Taïwan & $\begin{array}{c}\text { Séries de cas / } \\
n=2\end{array}$ & $\begin{array}{l}3 ? \\
5 ?\end{array}$ & $?(2)$ & $?(2)$ & $?(2)$ & $?(2)$ \\
\hline Masthi (56) / Inde & $\begin{array}{c}\text { Séries de cas / } \\
n=2\end{array}$ & $?$ & $?$ & $?$ & $?$ & $?$ \\
\hline $\begin{array}{l}\text { O'Malley (13) / États- } \\
\text { Unis }\end{array}$ & $\begin{array}{c}\text { Rapport de cas } \\
\qquad / \mathrm{n}=1\end{array}$ & $27 \mathrm{~F}$ & Perçage & $?$ & ++ & $?$ \\
\hline $\begin{array}{l}\text { Orwitz (57) / États- } \\
\text { Unis }\end{array}$ & $\begin{array}{c}\text { Rapport de cas } \\
/ \mathrm{n}=1\end{array}$ & $79 \mathrm{H}$ & Infection & $?$ & $?$ & $?$ \\
\hline $\begin{array}{l}\text { Percy (58) / États- } \\
\text { Unis }\end{array}$ & $\begin{array}{c}\text { Séries de cas / } \\
n=1\end{array}$ & $?$ & $?$ & + & $?$ & $?$ \\
\hline $\begin{array}{l}\text { Quackenbush (59) / } \\
\text { États-Unis }\end{array}$ & $\begin{array}{c}\text { Rapport de cas } \\
/ \mathrm{n}=1\end{array}$ & $44 \mathrm{~F}$ & Blessure & $?$ & $?$ & + \\
\hline $\begin{array}{l}\text { Rushdy (20) / } \\
\text { Royaume-Uni }\end{array}$ & $\begin{array}{c}\text { Rapport de } \\
\text { surveillance / } \\
\mathrm{n}=5\end{array}$ & $?$ & $?$ & $?$ & $?$ & $?$ \\
\hline Shimoni (15) / Israël & Rapport de cas & $34 \mathrm{H}$ & $?$ & $?$ & $?$ & + \\
\hline
\end{tabular}




\begin{tabular}{l|c|c|c|c|c|c} 
& $/ \mathrm{n}=1$ & & & \\
\hline $\begin{array}{l}\text { Srigley (60) / } \\
\text { Canada }\end{array}$ & $\begin{array}{c}\text { Rapport de cas } \\
/ \mathrm{n}=1\end{array}$ & $78 \mathrm{~F}$ & Blessure & $?$ & $?$ & -
\end{tabular} \\ ${ }^{1} \mathrm{H}$ (homme), $\mathrm{F}$ (femme), ? (inconnu / non indiqué) \\ 2 ? (inconnu / non indiqué) \\ $3++(3$ doses ou plus $),+(1$ ou 2 doses $), ?$ (nombre exact de doses non indiqué) \\ ${ }^{4}++(>0,1 \mathrm{Ul} / \mathrm{mL}),+(0,01-0,09 \mathrm{Ul} / \mathrm{mL}), ?=$ non mesuré \\ ${ }^{5}+$ (en vie au moment de quitter l'hôpital), - (décédé), ? (inconnu / non indiqué) \\ ${ }^{6}$ Études décrites dans le tableau (p. 127). On a obtenu les études initiales dans la mesure du possible (Long, Hall, Boyd, Boyer, Christensen). \\ Les autres études n'étaient pas indexées dans PubMed, MEDLINE et EMBASE. On a communiqué avec le Journal of the American Medical \\ Association (JAMA) pour obtenir la liste de référence initiale qui a été exclue du manuscrit publié en raison de sa longueur, mais aucun \\ exemplaire n'a été archivé. Les cas résumés dans le présent document sont de Moss et Hedrick. \\ UDI = Utilisateur de drogues injectables \\ IS = immunosuppression
}

\title{
Discussion
}

À la connaissance des auteurs, il s'agit du premier examen systématique qui évalue l'occurrence du tétanos chez des personnes ayant déjà été vaccinées. Depuis 1946, au moins 359 cas de tétanos ont été décrits chez des personnes ayant déjà été vaccinées et parmi celles dont les résultats ont été signalés, on a observé un taux de survie de $85,7 \%$, parmi lesquels peu de cas ont signalé des déficiences résiduelles au moment de quitter l'hôpital. Chez les cas ayant signalé le nombre de doses du vaccin comprenant l'anatoxine tétanique déjà reçues par personne, la gravité clinique de la maladie semblait être moindre que celle observée chez les personnes ayant reçu un moins grand nombre de doses antérieures (même si cela n'a pas pu être étudié de façon systématique en raison du faible nombre de décès et de cas symptomatiques au moment de quitter l'hôpital).

Une analyse précédente a révélé une relation similaire entre le nombre de doses et la gravité clinique. Un examen de 175 cas de tétanos signalés dans le cadre de la surveillance de routine entre 1984 et 2000 en Angleterre et au Pays de Galles a révélé que la gravité clinique de la maladie était plus importante chez les personnes sans antécédents d'immunisation (même si cela n'a pas atteint une signification statistique $(p=0,068))(20)$.

Les explications possibles de l'occurrence du tétanos clinique dans le cadre d'une immunisation passée pourraient comprendre : l'affaiblissement de l'immunité dérivée du vaccin, l'échec du vaccin, la présence d'une immunodéficience non reconnue entraînant une réponse immunitaire sous le seuil optimal pour la vaccination active ou un entreposage compromis des vaccins et une manipulation des vaccins entraînant la réduction de l'immunogénicité du vaccin. Par ailleurs, le fardeau de l'exotoxine tétanique peut dépasser la réponse immunitaire d'une personne qui peut également être influencée par des facteurs qui causent une immunosuppression, comme les maladies chroniques ou les médicaments.

Cet examen comprend les limites suivantes : l'incapacité à évaluer la fréquence de ce phénomène s'explique par l'absence d'un dénominateur, la possibilité de biais de publication et les données incomplètes ( $p$. ex. la survie). En outre, les limites inhérentes aux études de cas et aux rapports de surveillance comprennent les suivantes : la collecte de renseignements sur la source (p. ex. biais de rappel si les données sont autodéclarées, qualité et constance des données si elles proviennent d'une base de données, sous-déclaration ou capacité à déceler tous les cas dans un système de surveillance) et le manque d'uniformité de la définition clinique pour le tétanos dans les rapports de cas a créé des difficultés d'interprétation des données.

Néanmoins, cette étude apporte une contribution importante, car elle constitue possiblement le premier examen systématique résumant les caractéristiques du tétanos dans les cas précédemment immunisés par l'anatoxine tétanique. Les autres points forts de cette étude sont les suivants : la méthodologie systématique utilisée pour déterminer les études pertinentes et l'inclusion d'articles de nombreux pays, d'études de 1946 à 2013 et d'études menées dans les deux langues.

L'atténuation de la gravité de la maladie dans les hôtes vaccinés indique une possibilité de sous-déclaration si la personne ne se présente pas pour obtenir des soins médicaux, ainsi qu'une possibilité de retard de diagnostic, 
même si cela n'était pas toujours décrit dans la liste des articles inclus. Cette situation a des conséquences importantes pour la surveillance des maladies évitables par la vaccination et pour la pratique clinique.

Les futures recherches pourraient être axées sur la compréhension des répercussions du tétanos chez les personnes ayant déjà reçu une vaccination à l'anatoxine tétanique et si la maladie est attribuable à un affaiblissement de l'immunité ou à l'échec de l'immunisation, sur le moment optimal pour administrer les doses de rappel du vaccin comprenant l'anatoxine tétanique et sur des recherches plus approfondies sur le seuil d'anticorps contre le tétanos et leur rôle dans la détermination de l'immunité au tétanos.

\section{Conclusions}

Le tétanos est une maladie rare, mais potentiellement létale et la bactérie Clostridium tetani est omniprésente dans l'environnement. Une série de vaccination primaire complète et des doses de rappel appropriées ne confèrent manifestement pas une immunité à tous les vaccinés. Toutefois, le taux de survie est élevé chez les personnes ayant déjà reçu des doses documentées du vaccin comprenant l'anatoxine tétanique. Les cliniciens doivent conserver un taux élevé de suspicion clinique pour le tétanos lorsque les symptômes cliniques semblent l'indiquer, peu importe les antécédents de vaccination.

\section{Remerciements}

Les auteurs tiennent à remercier sincèrement les services de la bibliothèque de Santé publique Ontario, en particulier Beata Pach, pour son aide lors de l'analyse systématique et la récupération de la documentation et $D^{\text {re }}$ Shelley Deeks pour ses commentaires utiles sur une ébauche antérieure du manuscrit.

\section{Conflit d'intérêts}

II n'y a aucun conflit d'intérêts à déclarer.

\section{Financement}

Aucun financement n'a été reçu pour cette étude.

\section{Références}

(1) American Public Health Association. Tetanus. In: Heymann DL, editor. Control of communicable diseases manual, 19th Ed. Washington, D. C. : American Public Health Association; 2008. 602-8.

(2) Agence de la santé publique du Canada. Le tétanos. 2012 [mis à jour le 2012-11-23; consulté le 2013-05-03]; Accès : http://www.phac-aspc.gc.ca/im/vpd-mev/tetanus-fra.php

(3) Agence de la santé publique du Canada. Anatoxine tétanique. 2012. In : Guide canadien d'immunisation [en ligne]. Agence de la santé publique Canada. Accès : http://www.phac-aspc.gc.ca/publicat/cig-gci/p04-tet-fra.php

(4) Tiwari T, Clark TA, Messonnier NE, Thomas CG. Tetanus surveillance - United States, 2001-2008. Morbidity and Mortality Weekly Report. 2011; 60(12):365-9.

(5) Plotkin SA. Correlates of vaccine-induced immunity. Clinical Infecious Diseases. 2008; 47:401-9.

(6) Plotkin SA. Correlates of protection induced by vaccination. Clinical and Vaccine Immunology. 2010; 17(7):1055-65.

(7) McComb JA. The prophylactic dose of homologous tetanus antitoxin. New England Journal of Medicine. 1964; 270(4):175-8.

(8) Abrahamian FM, Pollack Jr. CV, LoVecchio F, Nanda R, Carlson RW. Fatal tetanus in a drug abuser with "protective" antitetanus antibodies. Journal of Emergency Medicine. 2000; 18(2):189-93.

(9) Atabek ME, Pirgon O. Tetanus in a fully immunized child. Journal of Emergency Medicine. 2005; 29(3):345-6.

(10) König K, Ringe H, Dorner BG, Diers A, Uhlenberg B, Müller D, et al. Atypical tetanus in a completely immunized 14year-old boy. Pediatrics. 2007; 120:e1355-8. 
(11) Livorsi DJ, Eaton M, Glass J. Generalized tetanus despite prior vaccination and a protective level of anti-tetanus antibodies. American Journal of the Medical Sciences. 2010; 339(2):200-1.

(12) Luisto M, livanainen M. Tetanus of immunized children. Developmental Medicine and Child Neurology. 1993; 35:34658.

(13) O'Malley CD, Smith N, Braun R, Prevots DR. Tetanus associated with body piercing. Clinical Infecious Diseases. 1998; 27(5):1343-4.

(14) Passen EL, Andersen BR. Clinical tetanus despite a "protective" level of toxin-neutralizing antibody. Journal of the American Medical Association. 1986; 255(9):1171-3.

(15) Shimoni Z, Dobrousin A, Cohen J, Pitlik S. Tetanus in an immunised patient. British Medical Journal. $1996 ; 319: 1049$.

(16) Berger SA, Cherubin CE, Nelson S, Levine L. Tetanus despite preexisting antitetanus antibody. Journal of the American Medical Association. 1978; 240(8):769-70.

(17) Crone NE, Reder AT. Severe tetanus in immunized patients with high anti-tetanus titers. Neurology. $1992 ; 42: 761-4$.

(18) Peterson H-I. A case of tetanus in spite of active toxoid prophylaxis. Acta Chirurgica Scandinavica. 1965; 129:235-7.

(19) Vieira BI, Dunne JW, Summers Q. Cephalic tetanus in an immunized patient. Medical Journal of Australia. 1986; 145(Aug 4/18):156-7.

(20) Rushdy AA, White JM, Ramsay ME, Crowcroft NS. Tetanus in England and Wales, 1984-2000. Epidemiology and Infection. 2003; 130:71-7.

(21) Pascual FB, McGinley EL, Zanardi LR, Cortese MM, Murphy TV. Tetanus surveillance - United States, $1998-2000$. Morbidity and Mortality Weekly Report. 2003; 52(SS03):1-8.

(22) Bardenheier B, Prevots DR, Khetsuriani N, Wharton M. Tetanus surveillance - United States, 1995-1997. Morbidity and Mortality Weekly Report. 1998; 47(SS-2):1-13.

(23) Newton-John HF. Tetanus in Victoria, 1957-1980. Review of 106 patients managed in one hospital. Medical Journal of Australia. 1984; 140(4):194-200.

(24) van den Hoek A, Sonder GJ, Scholing M, Gijselaar DB, van Binnendijk RS. Two cases of mild IgM-negative measles in previously vaccinated adults, the Netherlands, April and July 2011. Eurosurveillance. 2011; 16(48):1-3.

(25) Rota JS, Hickman CJ, Sowers SB, Rota PA, Mercader S, Bellini WJ. Two case studies of modified measles in vaccinated physicians exposed to primary measles cases: high risk of infection but low risk of transmission. Journal of Infectious Diseases. 2011; 204(Suppl. 1):S559-63.

(26) Chaves SS, Zhang J, Civen R, Watson BM, Carbajal T, Parella D, et al. Varicella disease among vaccinated persons: clinical and epidemiological cases 1997-2005. Journal of Infectious Diseases. 2008; 197(Suppl. 2):S127-31.

(27) Dyce O, Bruno JR, Hong D, Silverstein K, Brown MJ, Mirza N. Tongue piercing: the new "rusty nail". Head \& Neck. 2000; 22(7):728-32.

(28) Otero-Maldonado M, Bosques-Rosado M, Soto-Malavé R, Deliz-Roldán B, Bertrán-Pasarell J, Vargas Otero P. Tetanus is still present in the 21st centruy: case report and review of literature. Asociación Médica de Puerto Rico. 2011; 103(2):41-7.

(29) Aydin-Teke T, Bayhan GI, Afsariar CE, Oz FN, Akansel AR, Tanir G. A report of two pediatric tetanus cases. Turkish Journal of Pediatrics. 2011; 53(4):437-40.

(30) Boyd JSK. Tetanus in the African and European theatres of war: 1939-1945. Lancet. 1946; 1(6387):113-9.

(31) Boyer J, Corre-Hurst L, Sapin-Jaloustre H, Tissier M. Le tétanos en milieu urbain. Conditions d'apparition - déductions prophylactiques. La Presse Medicale. 1953; 61(34):701-3.

(32) Coniglione T, Onarecker C, Pryor T. Elevated antitoxin titers in a man with generalized tetanus. Journal of Family Practice. 1997; 44(3):299-303.

(33) de La Chapelle A, Lavabre O, Pinsard M, Delamonica J, Relyveld EH. Tetanus in a renal transplant recipient exhibiting the presence of circulating antitetanus antibodies determined by ELISA. Biomedicine \& Pharmacotherapy. 2002; 56(4):208-10.

(34) Faust RA, Vickers OR, Cohn I. Tetanus: 2449 cases in 68 years at a Charity Hospital. Journal of Trauma. 1976; 16(9):704-12.

(35) Fiorillo L, Robinson JL. Localized tetanus in a child. Annals of Emergency Medicine. 1999; 33(4):460-3.

(36) Hall WW. The U. S. Navy's war record with tetanus toxoid. Ann Intern Med. 1948; 28(2):298-308.

(37) Hedrick EC. Tetanus: two cases in immunised persons. California Medicine. 1953; 79(1):49-50.

(38) Lodha R, Sareen A. Tetanus in immunized children. Indian Pediatrics. 2000; 37(2):223-4. 
(39) Long AP. Immunization to tetanus. 20092009 Jun 1. In: Medical Science Publication No 4 [en ligne]. U. S. Army Medical Department. Accès : http://history.amedd.army.mil/booksdocs/korea/recad2/ch6-6.html

(40) Loscalzo IL, Ryan J, Loscalzo J, Sama A, Cadag S. Tetanus: a clinical diagnosis. American Journal of Emergency Medicine. 1995; 13(4):488-90.

(41) Spittle BJ, Pollock M, O'Donnell TV. Tetanus occurring in spite of active immunisation. New Zealand Medical Journal. 1973; 77(491):250-1.

(42) Bankole IA, Danesi MA, Ojo OO, Okubadejo NU, Ojini FI. Characteristics and outcome of tetanus in adolescent and adult patients. Journal of the Neurological Sciences. 2012; 15(323):201-4.

(43) Beltran A, Go E, Haq M, Clarke HB, Zaman M, Recco RA. A case of clinical tetanus in a patient with protective antitetanus antibody level. Southern Medical Journal. 2007; 100(1):83.

(44) Bunch TJ, Thalji MK, Pellikka PA, Aksamit TR. Respiratory failure in tetanus: case report and review of a 25-year experience. Chest. 2002; 122(4):1488-92.

(45) Christensen NA, Thurber DL. Clinical experience with tetanus: 91 cases. Proceedings of the Staff Meetings Mayo Clinic. 1957; 32(7):146-58.

(46) Culbertson TA, Kalliainen LK, Buchele BA. Tetanus and the plastic surgeon. Annals of Plastic Surgery. 2004; 53(2):1625 .

(47) deSouza CE, Karnad DR, Tilve GH. Clinical and bacteriological profile of the ear in otogenic tetanus: a case control study. Journal of Laryngology and Otology. 1992; 106(12):1051-4.

(48) Earis JE, Hillis AN, Macaulay MB. Tetanus: an unusual source and site of infection. Journal of Infection. 1983; 7(1):723.

(49) Edsall G. Specific prophylaxis of tetanus. Journal of the American Medical Association. 1959; 171(4):125-35.

(50) Ferris DMS. A case of tetanus modified by previous immunization, presenting as a psychiatric condition. Nursing Times. 1968; 64(34):1139-40.

(51) Geeta MG, Krishnakumar P, Mathews L. Intrathecal tetanus immunoglobulins in the management of tetanus. Indian Journal of Pediatrics. 2007; 74(1):43-5.

(52) Hahn BJ, Erogul M, Sinert R. Case report of tetanus in an immunized, healthy adult and no point of entry. Journal of Emergency Medicine. 2004; 27(3):257-60.

(53) Henderson SO, Mody T, Groth DE, Moore JJ, Newton E. The presentation of tetanus in an emergency department. Journal of Emergency Medicine. 1998; 16(5):705-8.

(54) Iqbal S, ul Iman N, ur Rahman S, Haroon M, Zaheer Z, ur Rahman N, et al. Trends of tetanus patients in North of Pakistan. Journal of Medical Sciences (Peshawar). 2012; 20(2):90-3.

(55) Lee H-C, Ko W-C, Chuang Y-C. Tetanus of the elderly. Journal of Microbiology, Immunology and Infection. 2000; 33(3):191-6.

(56) Masthi NRR, Bharat G, Aswini, Chitra, Arul PPM. A clinico epidemiological study of tetanus cases admitted to epidemic disease hospital, Bangalore. Indian Journal of Public Health. 2008; 52(4):210-11.

(57) Orwitz JI, Galetta SL, Teener JW. Bilateral trochlear nerve palsy and downbeat nystagmus in a patient with cephalic tetanus. Neurology. 1997; 49(3):894-5.

(58) Percy AS, Kukora JS. The continuing problem of tetnaus. Surgery, Gynecology and Obstetrics. 1985; 160(4):307-12.

(59) Quackenbush P, Tuorinsky S, Rabb R. Tetanus diagnosis sometimes elusive. Nurse Practitioner. 2003; 28(11):50-3.

(60) Srigley JA, Haider S, Johnstone J. A lethal case of generalized tetanus. Canadian Medical Association Journal. 2011; 183(9):1045-48. 